\title{
THE EFFECTS OF FIRE ON VIRGIN NORTHERN MIXED GRASSLAND AT CUSTER NATIONAL MONUMENT
}

\author{
Jane H. Bock \\ Maureen O'Shea Stone \\ E.P.O. Biology Department, Box 334 \\ University of Colorado \\ Boulder
}

Our second season of field work at the Battlefield ended in July, 1985. During this second season, we resampled the 25 study plots we established in 1984 (see Eighth Annual Report). In the wildfire of August, 1983, 20 of our study plots were burned (the experimental plots), and 5 were not (the control plots). The study plots are indicated in the sketch map, Figure 1. An excavation, approximately $1 \mathrm{~m}^{2}$, was made by the amateur archeologists visiting the site. No further incidents of this sort are anticipated for the duration of the study.

We sampled vegetation on all plots 2 by means of vegetation transects and quadrats. All plot measured $20 \times 20 \mathrm{~m}^{2}$. Transects on the plots were spaced $5 \mathrm{~m}$ apart, and sampling was carried out at $0.5 \mathrm{~m}$ intervals. At each sampling point we recorded species identification, height of the plant, and phenological state of the plant. If no living plants were present, this was noted. Twenty $50 \times 50$ $\mathrm{cm}$ quadrats were located inside the borders of each plot at $4 \mathrm{~m}$ intervals. Within each quadrat, we recorded all species present, numbers of individuals of each species, and a cover-sociability index number for each species. Litter, bare ground, and dead grass also were given cover values. All woody species were censused on all study piots. All living woody plants were identified to species, their total heights and canopy widths recorded, their phenological states and general health noted. As in the first year, each study plot was photographed from the coordinate stake which gave that plot its name. The camera was aimed diagonally across the study plot in each case. On all 25 plots, two $0.2 \mathrm{~m}$ samples of above-ground vegetation were clipped as in 1985. Samples were processed at the C.U. laboratory.

Several dozen photos of the Battlefield have been examined. They were taken through the years since the Battle (1876). We have rephotographed exactly several sites used in more recent ones which are commercially available. The study of old photos has told us a great deal about the nature of the Battlefield at the time of the Battle and how it has changed since. We are concluding that (1) the 1983 fire changed the appearance of the Battlefield from its pre-burn condition. We also feel that it may now be less like the condition of the Battlefield at the time of the Battle in 1876 than it was prior to the 1983 fire. The Battlefield offers a fine example of Northern Mixed Grass Prairie, and its natural values, supplemental perhaps to its historic ones, should not be undervalued by the National Park Service.

Our work of the checklist of the Monument is progressing. We have found over 


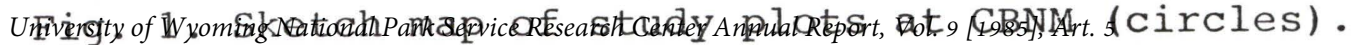
$\mathrm{E}=$ experimental plots. C=Control plots. Grid numbers are from the NPS survey.

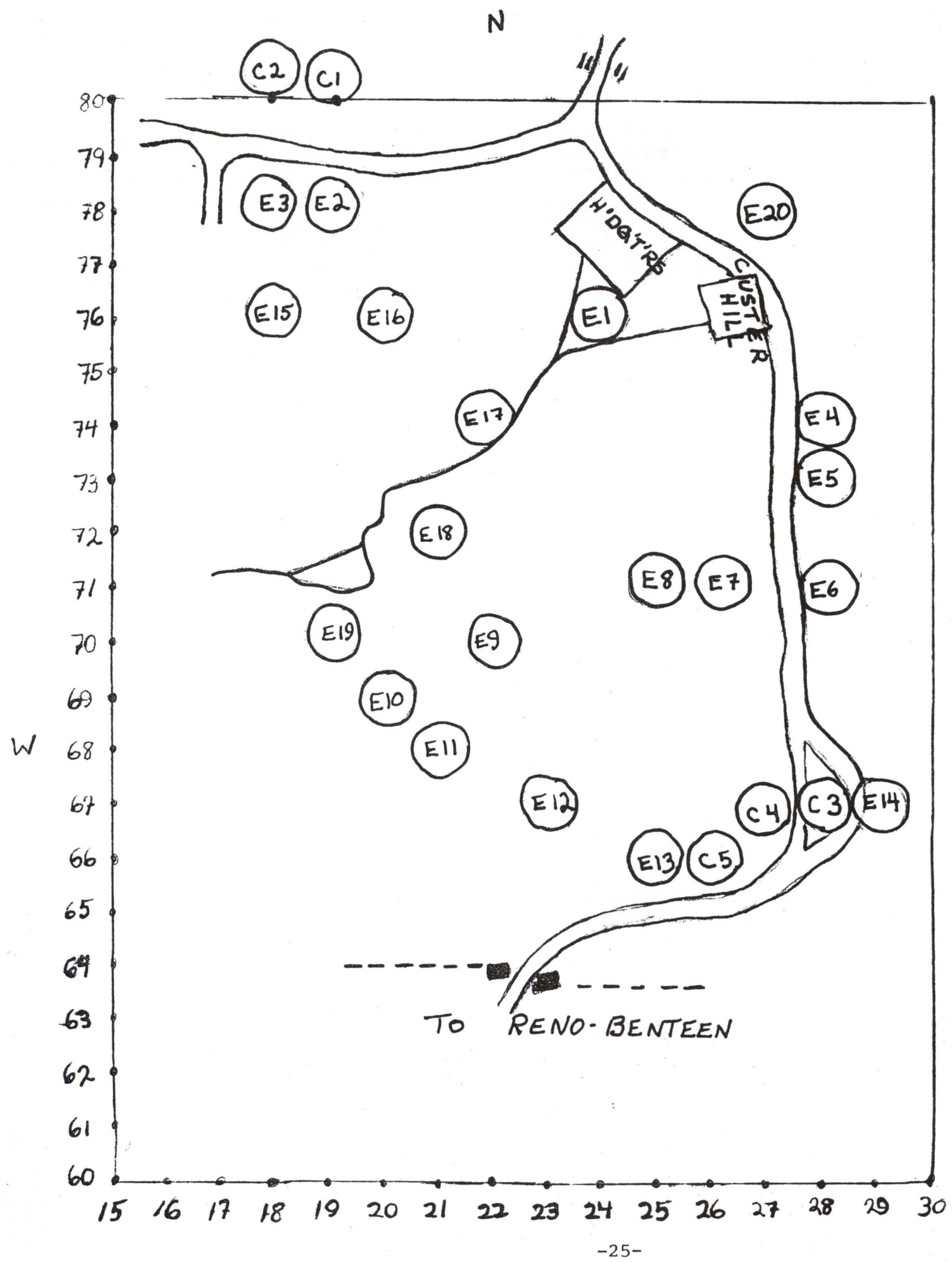


one-hundred species, and we estimate that this represents at least $90 \%$ of the Monument's flora. The checklist (Figure 2) is to be considered tentative at present because all identifications have not been confirmed by the Herbarium staff at Montana State University where we are depositing our vouchers.

This year we initiated a study of bird responses to the burn. There appears to be a strong correlation between vegetation structure and bird distributions. The fire decimated big sage (Artemisia tridentata). The only bird which occurred in any abundance on the burned areas was the Western Meadowlark. Brewer's Sparrows, Lark Sparrows, Lark buntings, and Grasshopper Sparrows all chose unburned sites with dense stands of sagebrush. Because other workers have found sage bird communities to vary greatly from year to year, we will repeat the bird observations in 1986, the last year of the study. 
Figure 2. Tentative checklist of the flora, Custer Battlefield National Monument

Yucca glauca

Rhus trilobata

Musineon divaricatum

Toxicodendron rydbergii

Ascepias speciosa

Achillea millefolium

Antennaria corymbosa

Antennaria parvifolia

Amica sororia

Artemisia cana

Artemisia frigida

Artemisia filifolia

Artemisia tridentata

Aster scopulorum

Centaura repens

Cirsium arvense

Cirsium undulatum

Crepis occidentalis

Echinacea angustifolia

Erigeron bellidiastrum

Erigeron ochroleucus

Grindelia squarrosa

Haplopappus langinosus

Helianthus sp.

Heterotheca villosa

Lactuca serriola

Lygodesmia junca

Machaeranthera pinnatifida

Microseris nutans

Ratibida columnifera

Senecio plattensis

Solidago nemoralis

Taraxicum officinale

Lappula redowski

Lithospermum incisum

Alyssum alyssoides

Alyssum desertorum

Draba brachycarpa

Erysimum asperum

Lepidium perfoliatum

Lesquerella ludoviciana

Sisymbrium altissimum

Thlaspi arvense

Corypanth sp.

Opuntia polyacantha

Lonicera tatarica

Symphoricarpos occidentalis

Seratoides lanata
Yucca

Skunkbrush

Poison Ivy

Showy Milkweed

Yarrow

Pussytoes

Pussytoes

Sweet Sage

Pasture Sage

Silver worm wood

Big Sagebrush

Star Thistle

Canada Thistle

Wavy-leaved Thistle

W. Hawksbeard

Purple Coneflower

Daisy Fleabane

Gum weed

Sunflower

Golden Aster

Prickly Lettuce

Skeleton Plant

False Dandelion

Coneflower

Groundsel

Goldenrod

Common Dandelion

Beggers Tick

Cleft Gromwell

Alyssum

Alyssum

W. Wallflower

Clasping Peppergrass

Bladderpod

Tumbling Mustard

Pennycress

Puncushion Cactus

Prickly Pear

Honeysuckle

Snowberry

Winterfat
Agavaceae

Anacardiaceae

Apiaceae

Apocynaceae

Asclepidaceae

Asteraceae

Asteraceae

Asteraceae

Asteraceae

Asteraceae

Asteraceae

Asteraceae

Asteraceae

Asteraceae

Asteraceae

Asteraceae

Asteraceae

Asteraceae

Asteraceae

Asteraceae

Asteraceae

Asteraceae

Asteraceae

Asteraceae

Asteraceae

Asteraceae

Asteraceae

Asteraceae

Asteraceae

Asteraceae

Asteraceae

Asteraceae

Asteraceae

Boraginaceae

Boraginareae

Brassicaceae

Brassicaceae

Brassicaceae

Brassicaceae

Brassicaceae

Brassicaceae

Brassicaceae

Brassicaceae

Cactaceae

Cactaceae

Caprifoliaceae

Caprifoliaceae

Chenopodiaceae 
Sarcobatus vermiculatus

Tradescantia bractata

Carex filifolium

Scirpus acutus

Eleagnus commutata

Shepherdia argentea

Euphorbia robusta

Astragalus aboriginum

Astragalus agrestis

Astragalus atropubescens

Astragalus crassicarpus

Astragalus drummondii

Astragalus hyalinus

Glycymiza lepidota

Lupinus argenteous

Medicago sativa

Melilotus officinalis

Oxytropis sericea

Petalostemon candidum

Petalostemon purpureum

Psoralea argophylla

Psoralea esculenta

Psoralea tenuiflora

Thermopsis rhombifolia

Vicia americana

Ribes aureum

Phacelia linearis

Hypericum perforatum

Allium textile

Calochortus nuttallii

Leucocrinum montanum

Smilacina stellata

Zigadenus venenosus

Linum perenne

Linum sulcatum

Sphaeralcea coccinea

Fraxinus pennsylvanica

Epilobium sp.

Gaura coccinea

Oenothera laciniata

Oenothera albicaulis

Plantago patagonica

Agrostis hyemalis

Agropyron dasystachyum

Agropyron desortorum

Agropyron repens

Agropyron smithii

Agropyron spicatum

Aristida purpurea

Bouteloua curtipendula

Bouteloua gracilis
Greasewood

Spiderwort

Bullrush

Silverberry

Buffaloberry

Indian Milk Vetch

Wild Licorice

Lupine

Alfalfa

Yellow Sweet Clover

White Locoweed

Wt. Prairie Clover

Pl. Prairie Clover

Wild Turnip

Golden Banner

American Vetch

wild Current

St. Johns Wort

Wild Onion

Mariposa Lily

Sand Lilly

Death Camas

Blue Flax

Yellow Flax

Scarlet Mallow

Green Ash

Scarlet Gaura

Evening Primrose

Ticklegrass

Thickspike Wh. grass

Crested Wheatgrass

Quackgrass

W. Wheatgrass

Bluebunch Wh. grass

Purple Three-A wn

Side Oats Grama

Blue Grama
Chenopodiaceae

Commelinaceae

Cyperaceae

Cyperaceae

Eleagnaceae

Eleagnaceae

Euphorbiaceae

Fabaceae

Fabaceae

Fabaceae

Fabaceae

Fabaceae

Fabaceae

Fabaceae

Fabaceae

Fabaceae

Fabaceae

Fabaceae

Fabaceae

Fabaceae

Fabaceae

Fabaceae

Fabaceae

Fabaceae

Fabaceae

Grossulariaceae

Hydrophyllaceae

Hydrophyllaceae

Iilliaceae

Liliaceae

Lilliaceae

Liliaceae

Liliaceae

Linaceae

Linaceae

Malvaceae

Oleaceae

Onagraceae

Onagraceae

Onagraceae

Onagraceae

Plantaginaceae

Poaceae

Poaceae

Poaceae

Poaceae

Poaceae

Poaceae

Poaceae

Poaceae

Poaceae 
Bromus inermis

Bromus japonicus

Bromus tectorum

Calamagrostis inexpansa

Calamovilfa longifolia

Hordeum jubatum

Koeleria macrantha

Poa compressa

Poa juncifolia

Poa pratensis

Stipa comata

Stipa viridula

Triticum aestivum

Collomia linearis

Phlox hoodii

Eriogonum pauciflorum

Rumex crispus

Delphinium nuttallianum

Rosa arkansana

Galium aparine

Galium obtusum

Populus deltoides

Comandra umbellata

Castilleja angustifolia

Orthocarpus luteus

Penstemon albidus

Penstemon eriantherus

Penstemon nitidus

Typha latifolia

Viola nuttalli

Smooth Brome
Japanese Brome
Cheat Grass
Northern Reedgrass
Prairie Sandreed
Foxtail Barley
Junegrass
Canada Bluegrass
Kentucky Bluegrass
Needle \& Thread Grass
Green Needlegrass
Wheat
Phlox
Curly Dock
Larkspur
Wild Rose
Cleavers
Smooth Galium
Plains Cottonwood
Bastard Toadflax
Indian Paintbrush
Owls Clover
Penstemon

Cat Tail

Nuttalls Violet
Poaceae

Poaceae

Poaceae

Poaceae

Poaceae

Poaceae

Poaceae

Poaceae

Poaceae

Poaceae

Poaceae

Poaceae

Poaceae

Polemoniaceae

Polemoniaceae

Polygonaceae

Polygonaceae

Ranunculaceae

Rosaceae

Rubiaceae

Rubiaceae

Salicaceae

Santalaceae

Scrophlariaceae

Scrophulariaceae

Scrophulariaceae

Scrophulariaceae

Scrophulariaceae

Typhaceae

Violaceae 